\title{
Myocardial injury after non-cardiac surgery: Time to shed the ignorance
}

\author{
E Coetzee, MB ChB, DA (SA), FCA (SA), MMed (Anaesth); B M Biccard, MB ChB, FFARCSI, FCA (SA), MMedSci, PhD \\ Department of Anaesthesia and Perioperative Medicine, Faculty of Health Sciences, Groote Schuur Hospital and University of Cape Town, \\ South Africa
}

Corresponding author: E Coetzee (ettiennec@gmail.com)

\begin{abstract}
Perioperative cardiovascular complications are common and place a significant burden on public healthcare systems. A large proportion of such complications are due to a new clinical entity, i.e. myocardial injury after non-cardiac surgery (MINS). It is important to understand MINS, its prognosis and management in the perioperative period. A literature review of MINS was done. MINS is defined as an elevated postoperative cardiac troponin level that was considered as resulting from myocardial ischaemia without evidence of a non-ischaemic cause for the troponin elevation. The perioperative milieu (surgical stress response, sympathetic activation, hypercoagulability, hypotension, bleeding, anaemia and pain) contributes to the pathophysiology of a relative myocardial hypoperfusion and ischaemia, which differentiates MINS from myocardial infarction in non-surgical patients. Globally, $>7 \%$ of adults $\geq 45$ years of age suffer MINS, with South African (SA) studies confirming similar event rates. More than $80 \%$ of MINS patients are asymptomatic for myocardial ischaemia, and therefore would not fulfil the universal definition of myocardial infarction, despite having a similar prognosis to those with the latter condition. Accurate diagnosis of MINS therefore relies on routine daily postoperative cardiac troponin surveillance for $48-72$ hours postoperatively in patients with a $>5 \%$ risk of major perioperative cardiovascular complications. This approach is cost-effective in SA. One in 10 patients with MINS dies within 30 days of surgery, and 1 in 5 develops major cardiovascular complications. Short- and long-term mortality could be improved by simple treatment strategies, including cardiovascular therapy intensification, and by ensuring aspirin use and statin therapy. All recommendations promote the involvement of a multidisciplinary team. MINS is a common, serious perioperative cardiovascular complication with public healthcare implications that has been underappreciated in SA. A multidisciplinary approach with simple treatment strategies should be adopted.
\end{abstract}

S Afr Med J 2018;108(6):464-467. DOI:10.7196/SAMJ.2018.v108i6.13346

\section{Case vignette}

A clinical entry, after specialist referral of a patient who experienced a postoperative troponin elevation, reads as follows: 'Thank you for the referral. Reason for referral: postoperative troponin elevation following total hip replacement. According to the history, the patient was asymptomatic and comfortable in the ward. No ischaemic symptoms or electrocardiogram [ECG] changes noted. Vital signs were within normal limits. Assessment: isolated troponin elevation, expected in this patient population and not clinically significant. Recommendation: review patient for symptoms, no specific therapy required.'

After an uneventful hospital stay, the patient was discharged on the 4 th postoperative day, but died 5 days later at home.

The global surgical volume has increased to $>300$ million procedures per annum. ${ }^{[1]}$ Cardiovascular complications contribute to one-third of major perioperative morbidity and mortality, with perioperative myocardial infarction and myocardial injury after non-cardiac surgery (MINS) accounting for 39\% of all deaths within 30 days of non-cardiac surgery. ${ }^{[2]}$ These deaths are distributed equally between patients who sustain a postoperative myocardial infarction and MINS. ${ }^{[2]}$ The burden of perioperative cardiovascular complications, therefore, has a major public health importance. The paucity of clinicians with an appreciation of the importance of perioperative myocardial injuries and the associated prognosis, and the knowledge necessary to manage this public healthcare burden, needs to be addressed. ${ }^{[3]}$

\section{Methods}

A literature search on MINS in the perioperative period was conducted using MEDLINE and the following search terms: 'myocardial injury', 'noncardiac surgery', 'mortality', 'perioperative complications', 'treatment', 'prevention' and 'guidelines'. Additional reports were obtained by reviewing reference lists from the literature. Studies involving large cohorts with clear inclusion criteria and outcome data were reviewed. Reviews and smaller studies with local significance were also included.

\section{MINS}

MINS occurs when myocardial ischaemia-related cellular damage occurs in the perioperative period. It has been defined as an elevated cardiac troponin $\mathrm{T}$ ( $\mathrm{cTnT}$ ) level that was considered as resulting from myocardial ischaemia without evidence of a non-ischaemic cause of cTnT elevation (Table 1). ${ }^{[4]}$ The pathophysiology of MINS in surgical patients differs from that of myocardial infarction in medical (non-surgical) patients. ${ }^{[5]}$ Surgical patients are exposed to a unique environment that includes sympathetic activation, bleeding, anaemia, pain, hypotension and hypercoagulability during the perioperative period. Another distinctive clinical feature of MINS is that it most commonly presents within the first 3 postoperative days, strengthening its association with the early postoperative pathophysiological environment, in contrast to postoperative atherosclerotic plaque rupture that tends to occur more randomly during the weeks after surgery, and more closely resembles a traditional medical (non-surgical) myocardial infarction. ${ }^{[5]}$

\section{Incidence of MINS}

International data estimate that $>100$ million adults $\geq 45$ years of age undergo non-cardiac surgery per annum. ${ }^{[2]}$ In the recent Vascular Events In Non-cardiac Surgery Patients Cohort Evaluation (VISION) 
study non-cardiac surgical patients were studied for perioperative complications. ${ }^{[6]}$ More than 40000 patients were enrolled, of whom $7 \%$ experienced MINS. ${ }^{[4,7]}$ MINS is therefore a common perioperative complication, especially when one considers the nonselective nature of the VISION study's inclusion criteria: $\geq 45$ years of age and the need to spend a night in hospital following surgery, thereby providing a representative sample of perioperative outcomes in unselected surgical patients. Therefore, about 1 in 14 in-hospital patients $\geq 45$ years old, who undergo non-cardiac surgery, will experience MINS as a perioperative complication. Factors that increase the risk for experiencing MINS occur commonly in our surgical population. These include increasing age, patients with known cardiovascular disease or known risk factors for cardiovascular disease, and operations associated with an elevated risk (typically intermediate and major surgical severity, and all nonelective surgery) (Table 2). ${ }^{[4]}$

\section{Clinical presentation of MINS in the postoperative period}

To diagnose medical (non-surgical) myocardial infarction, the diagnostic criteria require documented elevated cardiac biomarkers and features of ischaemia (clinical, electrocardiographic or imaging). ${ }^{[8]}$ Current studies employ cTnT, as it is universally standardised. ${ }^{[9]}$ In contrast to medical myocardial infarction, clinical features of ischaemia are notoriously absent in surgical patients. Therefore, the diagnosis of MINS hinges on perioperative troponin elevation, and the exclusion of known non-ischaemic causes of perioperative troponin elevation, e.g. sepsis and pulmonary embolism. ${ }^{[4]}$ More than $93 \%$ of patients who experienced MINS, did not fulfil the diagnostic criteria for the

Table 1. MINS diagnostic criteria and hs-cTnT thresholds independently associated with 30-day mortality ${ }^{[7]}$

MINS diagnostic criteria (only one required)

Any peak hs-cTnT $\geq 65 \mathrm{ng} / \mathrm{L}$

An absolute increase in hs-cTnT of $5 \mathrm{ng} / \mathrm{L}$, with peak cTnT 20 - $64 \mathrm{ng} / \mathrm{L}$ hs-cTnT thresholds associated with 30-day mortality

\begin{tabular}{lll}
\cline { 2 - 3 } hs-cTnT, ng/L & $\begin{array}{l}\text { Adjusted hazard } \\
\text { ratio }(\mathbf{9 5} \% \mathbf{C I})^{*}\end{array}$ & 30-day mortality, \% \\
\hline $14-<20$ & $9.1(3.8-22.1)$ & 1.1 \\
$20-<65$ & $23.6(10.3-54.1)$ & 3.0 \\
$65-<1000$ & $70.3(30.6-161.7)$ & 9.1 \\
$\geq 1000$ & $227.0(87.4-589.9)$ & 29.6
\end{tabular}

MINS = myocardial injury after non-cardiac surgery; hs-c TnT = high-sensitivity cardiac troponin $\mathrm{T} ; \mathrm{CI}=$ confidence interval.

Table 2. Perioperative risk factors for the development of MINS $^{[4]}$

\begin{tabular}{l}
\hline Risk factor \\
\hline Increasing age \\
Current atrial fibrillation \\
Diabetes \\
Hypertension \\
Congestive heart failure \\
Coronary artery disease \\
Peripheral vascular disease \\
Stroke \\
Decreasing renal function \\
Urgent or emergency surgery \\
MINS = myocardial injury after non-cardiac surgery.
\end{tabular}

universal definition of myocardial infarction; only 7\% presented with ischaemic symptoms and $15 \%$ had nonspecific ECG changes. ${ }^{[4,7]}$

The majority of troponin elevations occur within 48 hours of surgery, and the patient's prognosis is positively correlated with the peak troponin level. ${ }^{[4,7]}$ Studies of the high-sensitivity ${ }^{c T n T}$ (hs-cTnT) test found that a small absolute increase in hs-cTnT from the preoperative to the postoperative period has prognostic use (Table 1). ${ }^{[7]}$ Furthermore, observational data have shown that $~ 50 \%$ of patients demonstrate an early 24-hour postoperative window, where the troponin levels remain low before a subsequent rise in these levels, highlighting the need for serial troponin surveillance in the postoperative period. ${ }^{[10]}$ As most of the prognostically significant troponin elevations occur asymptomatically, it is inappropriate to depend on ECG changes or ischaemic symptomatology to alert a clinician to MINS, as this will result in the majority $(>80 \%)$ of diagnoses being missed. ${ }^{[4]}$

\section{Cardiac troponin $\mathrm{T}$ thresholds should be considered according to clinical context}

The diagnostic troponin thresholds for MINS differ from those used to diagnose medical patients with myocardial infarction. ${ }^{[4,7,8]}$ Before cardiac biomarkers are used in clinical practice, their diagnostic thresholds need to be determined by investigating specific reference populations. ${ }^{[8]}$ The clinical environment of surgical and medical patients differs, which influences the sensitivity and specificity of these tests for specific outcomes and therefore requires populationspecific investigation. ${ }^{[9]}$ The literature investigating different troponin assays in perioperative patients has provided assay-specific troponin levels that are of prognostic relevance in the surgical patient (i.e. independently associated with 30 -day mortality), ${ }^{[4,7]}$ but may not be typically associated with an adverse prognosis in the non-surgical patient (Table 1).

\section{Prognosis of surgical patients with MINS}

MINS has a 30 -day mortality of $10 \% \cdot{ }^{[4]}$ One in 10 patients with MINS dies, and 1 in 5 patients suffers a major cardiovascular complication (congestive cardiac failure, non-fatal cardiac arrest and stroke) within 30 days of surgery. ${ }^{[4]}$ Various investigators have reported similar outcomes, as demonstrated by two meta-analyses independently associating postoperative troponin elevation with long- and shortterm mortality. ${ }^{[3]}$ A recent study found that the incidence of MINS in a South African (SA) patient population was comparable with those of large international cohorts. ${ }^{[11]}$ The 1500 SA patients included in the VISION cohort also suggested findings that could be compared with those of other international cohorts, and showed no evidence of differences between clusters. ${ }^{[6,7]}$ It is therefore reasonable to assume that the SA patient population has a similar risk for MINS and outcomes after MINS to that in the literature.

Patients who develop MINS postoperatively, have prognostic risk factors independently associated with 30-day mortality (Table 3). ${ }^{[4]}$ A point system allows for prediction of the risk of 30-day mortality:

Table 3. Independent predictors of 30-day mortality in patients who experience MINS ${ }^{[4]}$

\begin{tabular}{ll}
\hline Independent predictors & $\begin{array}{l}\text { Adjusted odds ratio } \\
(\mathbf{9 5 \%} \mathbf{C I})^{*}\end{array}$ \\
\hline Age $\geq 75$ years & $2.06(1.33-3.37)$ \\
ST elevation or new LBBB & $3.96(1.54-9.14)$ \\
Anterior ischaemic changes on ECG & $2.33(1.42-3.70)$ \\
MINS = myocardial injury after non-cardiac surgery; & LBBB = left bundle branch block; \\
ECG = electrocardiogram; $\mathrm{CI}=$ confidence interval. & \\
${ }^{*}$ All $p<0.01$.
\end{tabular}


0 points $(5 \%), 1$ point $(10 \%), 2$ points $(20 \%)$, 3 points (30\%) and 4 points $(50 \%) .{ }^{[4]}$ The cTnT diagnostic thresholds required for MINS, along with the 30-day prognostic impact, are shown in Table 1 . The prognostically significant impact of MINS in the perioperative period is thus clear and therefore demands that clinicians understand the clinical entity of MINS and its associated diagnostic criteria, prognosis, prognostic markers and the need for active therapeutic intervention. The consultation example shown in the introduction of this article can therefore no longer be accepted.

\section{Proposed management of MINS}

The most recently published perioperative cardiovascular guidelines propose postoperative troponin surveillance for patients who have a $>5 \%$ risk of a perioperative major adverse cardiovascular event. The following criteria apply: ( $i$ ) any patient $\geq 65$ years of age; (ii) patients between 45 and 64 years of age with a revised cardiac risk index (RCRI) of $\geq 1$; or (iii) any patient with an elevated preoperative natriuretic peptide (including brain natriuretic peptide or $\mathrm{N}$-terminal probrain natriuretic peptide). ${ }^{[12]}$ The recent Canadian guidelines on $\mathrm{cTnT}$ surveillance and RCRI score are summarised in Table 4. As complications are common in the first week after MINS, we would advocate early intervention. ${ }^{[13]}$ Evidence from randomised controlled trials is lacking, but we expect this to change over the next few years. ${ }^{[3]}$ The Management of Myocardial Injury After Noncardiac Surgery Trial (MANAGE) (clinicaltrials.gov/ct2/show/NCT01661101) results are currently in press, but initial reports indicate improved primary endpoints. MANAGE evaluated the impact of anticoagulation on perioperative vascular complications after MINS. The final results will provide more insight into therapeutic options.

Current observational data suggest that simple therapeutic measures could improve outcomes once MINS occurs. The literature supports the use of postoperative aspirin administration and statin therapy to reduce 30-day mortality after MINS. ${ }^{[14]}$ Furthermore, intensification of therapy (defined as introducing or increasing any of the following cardiovascular drug groups: antiplatelet agents, beta-blockers, statins or angiotensin converting enzyme inhibitors) during the postoperative period has been shown to be associated with an improved 1-year survival. ${ }^{[15]}$ However, deciding on a specific therapy should be part of a multidisciplinary approach. The potential benefits of therapies should be weighed against potential risks on an individual basis. After initiation of treatment intensification, surveillance for additional prognostic information should continue (Table 3). ${ }^{[4]}$ Increased postoperative monitoring (i.e. continuous invasive haemodynamic monitoring and a high-care facility) and optimised myocardial oxygen balance (i.e. haemodynamic support, correction of anaemia and electrolyte disturbances) could be of additional benefit, but is currently only supported by recommendations. ${ }^{[13]}$ A pharmacoeconomic study showed that troponin surveillance in the SA context should be cost-effective for all patients $\geq 45$ years of age who undergo non-cardiac surgery, but only when combined with active intervention once MINS is diagnosed. ${ }^{[16]}$
An adapted proposed algorithm for MINS management is depicted in Fig. 1.

\section{Conclusion}

MINS commonly occurs in non-cardiac surgical patients $\geq 45$ years of age. Ten percent of patients who experience MINS die within 30 days of surgery. MINS can be easily diagnosed if routine, postoperative hs-cTnT surveillance is conducted, as recommended by current international guidelines, and it is likely to be cost-effective in SA. Once MINS is diagnosed, institution of simple therapies may improve outcome. Large studies are expected to provide more therapeutic insight in the near future. Screening and treatment of MINS need to be incorporated into routine postoperative surgical management.

Table 4. Postoperative troponin surveillance recommendations from the Canadian Cardiovascular Society Guidelines ${ }^{[12,17]}$

Daily postoperative cTnT surveillance for 48 - 72 hours if baseline risk $>5 \%$ for

cardiovascular death or non-fatal myocardial infarction at 30 days after surgery, including: $:^{[12]}$

Elevated preoperative NT-proBNP or BNP measurement

RCRI score $\geq 1^{*}$

Age 45 - 64 years, with significant cardiovascular disease*

Age $\geq 65$ years*

RCRI ${ }^{[17]}$

High-risk-type surgery (intraperitoneal, intrathoracic or supra-inguinal vascular)

Ischaemic heart disease

History of congestive heart failure

History of cerebrovascular disease

Insulin therapy for diabetes

Preoperative serum creatinine $>2.0 \mathrm{mg} / \mathrm{dL}(177 \mu \mathrm{mol} / \mathrm{L})$

$\mathrm{RCRI}=$ revised cardiac risk index; NT-proBNP $=\mathrm{N}$-terminal probrain natriuretic peptide; $\mathrm{BNP}=$ brain natriuretic peptide cTnT $=$ cardiac troponin T; $\mathrm{CI}=$ confidence interval.

*If preoperative NT-proBNP or BNP not available.

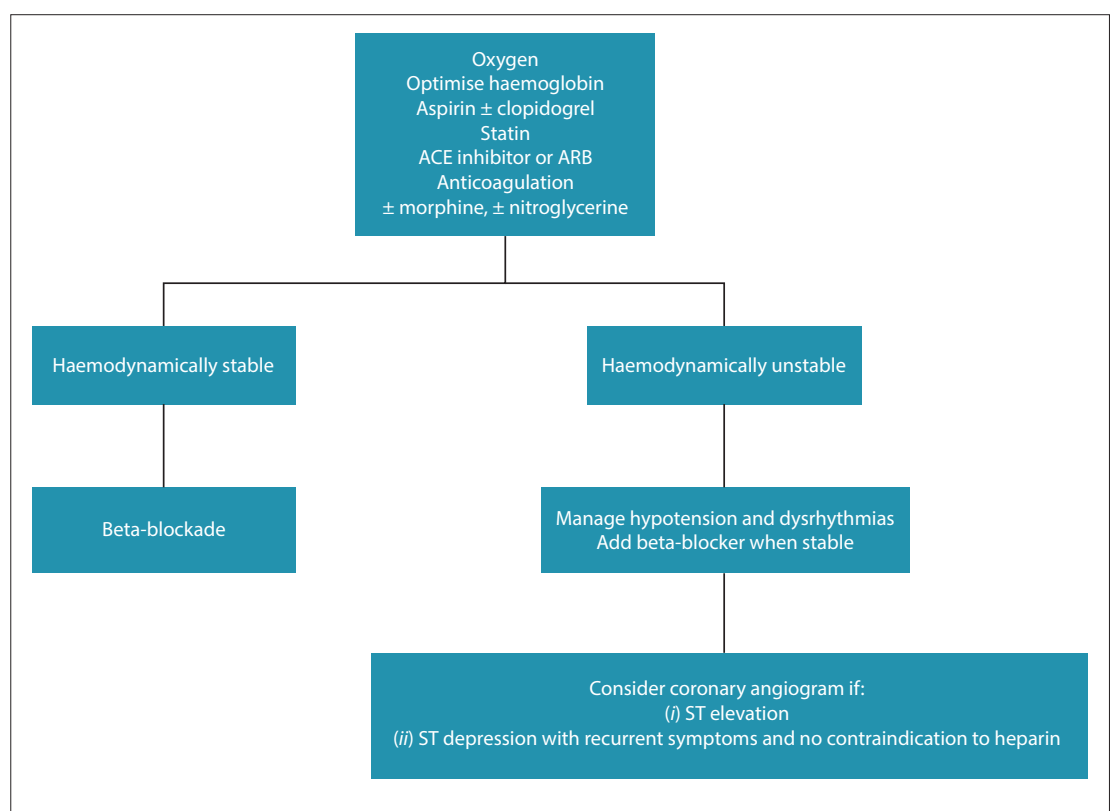

Fig. 1. Proposed algorithm for the management of patients with MINS. (Adapted from Biccard. ${ }^{[18]}$ MINS = myocardial injury after non-cardiac surgery; $A C E$ inhibitor = angiotensin converting enzyme inhibitor; $A R B=$ angiotensin receptor blocker.) 
Acknowledgement. None.

Author contributions. The draft article was produced by EC and all subsequent versions were edited and proofread by BMB and EC.

Funding. None.

Conflicts of interest. None.

1. Weiser TG, Haynes AB, Molina G, et al. Estimate of the global volume of surgery in 2012: An assessment supporting improved health outcomes. Lancet 2015;385(S11). https://doi.org/10.1016 An assessment support $6736(15) 60806-6$

2. Devereaux PJ, Sessler DI. Cardiac complications in patients undergoing major noncardiac surgery. N Engl J Med 2015;373(23):2258-2269. https://doi.org/10.1056/NEJMra1502824

3. Mauermann E, Puelacher C, Lurati Buse G. Myocardial injury after noncardiac surgery. Curr Opin Anaesthesiol 2016;29(3):403-412. https://doi.org/10.1097/ACO.0000000000000336

4. Botto F, Alonso-Coello P, Chan MTV, et al. Myocardial injury after noncardiac surgery. Anesthesiology 2014;120(3):564-578. https://doi.org/10.1097/ALN.0000000000000113

5. Biccard BM, Rodseth RN. The pathophysiology of peri-operative myocardial infarction. Anaesthesia 2010;65(7):733-741. https://doi.org/10.1111/j.1365-2044.2010.06338.x

6. Devereaux PJ, Chan MT, Alonso-Coello P, et al. Association between postoperative troponin levels and 30-day mortality among patients undergoing noncardiac surgery. JAMA 2012;307(21):2295-2304 https://doi.org/10.1001/jama.2012.5502

7. Devereaux PJ, Biccard BM, Sigamani A, et al. Association of postoperative high-sensitivity troponin levels with myocardial injury and 30-day mortality among patients undergoing noncardiac surgery. JAMA 2017;317(16):1642-1651. https://doi.org/10.1001/jama.2017.4360

8. Thygesen K, Alpert JS, Jaffe AS, et al. Third universal definition of myocardial infarction. Circulation 2012;126(16):2020-2035. https://doi.org/10.1161/CIR.0b013e31826e1058
9. Apple FS, Collinson PO. Analytical characteristics of high-sensitivity cardiac troponin assays. Clin Chem 2012;58(1):54-61. https://doi.org/10.1373/clinchem.2011.165795

10. Le Manach Y, Perel A, Coriat P, et al. Early and delayed myocardial infarction after abdominal aortic . Le Manach Y, Perel A, Coriat P, et al. Early and delayed myocardial infarction after abdominal aortic
surgery. Anesthesiology 2005;102(5):885-891. https://doi.org/10.1097/00000542-200505000-00004

11. Coetzee E, Biccard BM, Dyer RA, et al. Incidence of myocardial injury after non-cardiac surgery:
11. Anesthesiology 2005;102(5):85-81. http:/ doi.org/ $0.1097000052-20050500-0004$.

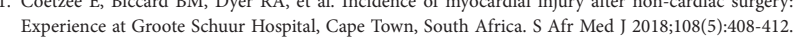
Experience at Groote Schuur Hospital, Cape Tow
https://doi.org/10.7196/SAMJ.2018.v108i5.12784

12. Duceppe E, Parlow J, MacDonald P, et al. Canadian Cardiovascular Society guidelines on perioperative cardiac risk assessment and management for patients who undergo noncardiac surgery. Can J Cardiol 2017;33(1):17-32. https://doi.org/10.1016/j.cjca.2016.09.008

13. Biccard BM. Detection and management of perioperative myocardial ischemia. Curr Opin Anaesthesiol 2014;27(3):336-343. https://doi.org/10.1097/ACO.0000000000000071

14. Devereaux $P$. Characteristics and short-term prognosis of perioperative myocardial infarction in patients undergoing noncardiac surgery. Ann Intern Med 2011;154(8):523-528. https://doi.org/10.7326/0003-4819154-8-201104190-00003

15. Foucrier A, Rodseth R, Aissaoui M, et al. The long-term impact of early cardiovascular therapy intensification for postoperative troponin elevation after major vascular surgery. Anesth Analg 2014:119(5):1053-1063. https://doi.org/10.1213/ANE.0000000000000302

16. Told $\mathrm{A}, \mathrm{Ry}$, L K Torborg A, Ryan L, Kar. $\mathrm{G}$, Bicard BM. The pharmacoeconomics of routine postoperative troponin 6rillance to prevent 2014;104(9):619-623. https://doi.org/10.7196/SAMJ.765

17. Lee TH, Marcantonio ER, Mangione CM, et al. Derivation and prospective validation of a simple index for prediction of cardiac risk of major noncardiac surgery. Circulation 1999;100(10):1043-1049. https://doi.org/10.1161/01.CIR.100.10.1043

18. Biccard B. Peri-operative myocardial infarction. S Afr J Anaesth Analg 2010;16(1):44-46. https://doi. org/10.1080/22201173.2010.10872633

Accepted 3 April 2018 